\title{
Perception and use of gait measures among physical therapists in South Korea
}

\author{
Ho Young Jang ${ }^{a}$, You Lim Kim ${ }^{b}$, Sung-jin Kim ${ }^{b}$, Tak Yong Yoon ${ }^{b}$, Kyung Hun Kimc, Ick Keun Ahn ${ }^{b}$, Suk Min Lee \\ ${ }^{a}$ Department of Physical Therapy, St. Paul's Hospital, The Catholic University of Korea, Seoul, Republic of Korea \\ bepartment of Physical Therapy, College of Health Science and Social Welfare, Sahmyook University, Seoul, Republic of Korea \\ 'Department of Physical Therapy, Gimcheon University, Gimcheon, Republic of Korea
}

Objective: The purpose of this study was to investigate the physical therapists' perception of the use of gait measures, the frequency of the gait measures used, and also to identify the barriers that limit the use of these assessment tools.

Design: Cross-sectional study.

Methods: Physical therapists from the Seoul, Gyeonggi area from March to July 2016 were included in the study. Over the course of 18 weeks, a cross-sectional study was conducted with a self-report questionnaire. A total of 700 questionnaires were distributed and 350 questionnaires $(50 \%)$ were collected, however with the exclusion of 140 questionnaires due to non-consent, a total of 210 questionnaires $(30 \%)$ were analysed.

Results: Out of the 10 standardized assessment tools, the therapists showed the highest perception for the timed up and go test (TUG $[\mathrm{n}=153,72.9 \%]$ ) and they also had high perception for the 10 meters walk test (10MWT $[\mathrm{n}=149,71.0 \%])$, and 6-minute walk test (6MWT $[\mathrm{n}=123,58.6 \%])$. The respondents answered that the TUG $(\mathrm{n}=116,55.2 \%), 10 \mathrm{MWT}(\mathrm{n}=100,47.6 \%)$, and 6MWT ( $\mathrm{n}=51,24.3 \%$ ) was used the most often. On the contrary, only four $(1.9 \%)$ therapists have used the Chedoke-McMaster stroke assessment and the Rivermead Mobility Index. The lack of time was considered as the most important barrier to the use of assessment tools in clinical practice.

Conclusions: Through this study, it has been shown that the domestic physical therapists used the TUG and the 10MWT mainly due to high recognition and evaluation status; however, the lack of time was the greatest impediment to the clinical application of the gait assessment tools.

Key Words: Gait, Measurement tools, Physical therapists

\section{Introduction}

Walking, which is defined as an activity that moves the limbs repeatedly to progress the body forward while maintaining postural stability, is considered as the most basic type of physical activity ability used in everyday life and social participation [1]. Musculoskeletal injuries or impairments of the nervous systems, such as stroke, traumatic brain injury, spinal cord injury, cerebral palsy, or Parkinson's disease may all cause gait pathologies, which may lead to difficulties in daily life practice and social participation, result- ing in poor quality of life [2].

Physical therapy is a method to improve physical mobility due to pathology through the use of several intervention methods, such as neurophysiological approaches (Bobath), biofeedback, electrical stimulation, physical fitness training, high-intensity therapy, repetitive task-specific training, gait training using visual cues, treadmill training plus body weight support and robotic-assisted body weight supported treadmill training [3-6].

For treatment to be effective, an accurate gait assessment of the patient should be prioritized. The assessment identi-

Received: 27 April, 2017 Revised: 6 June, 2017 Accepted: 7 June, 2017

Corresponding author: Suk Min Lee

Department of Physical Therapy, College of Health Science and Social Welfare, Sahmyook University, 815 Hwarang-ro, Nowon-gu, Seoul 01795, Republic of Korea Tel: 82-2-3399-1632 Fax: 82-2-3399-1639 E-mail: leesm@syu.ac.kr

(c) This is an Open-Access article distributed under the terms of the Creative Commons Attribution Non-Commercial License (http://creativecommons.org/licens es/by-nc/4.0) which permits unrestricted non-commercial use, distribution, and reproduction in any medium, provided the original work is properly cited.

Copyright $@ 2017$ Korean Academy of Physical Therapy Rehabilitation Science 
fies the cause of the gait impairment, assists to establish a treatment plan, and is used as an outcome measure. It provides information needed to educate patients and their caregivers, thereby gaining credibility. It is also a resource for sharing information among rehabilitation specialists and is an important part of evidence-based practice [7-9]. The standardized gait assessment tools currently being used include functional performance tests such as the 10 meters walk test (10MWT), 6-minute walk test (6MWT), timed up and go test (TUG), or motor assessment scale (MAS) such as the walking item, dynamic gait index (DGI), rivermead mobility index (RMI), functional ambulation classification (FAC), functional independent measure (FIM), ChedokeMcMaster stroke assessment, as well as other tools that are ordinal scale-based or instrumented measurement tools that produce kinetic and kinematic data [10-12].

Several evaluation tools such as these are currently being utilized for research or clinical evaluation purposes [13], and although having an accurate understanding and information about the assessment tools may serve as a stable basis for therapeutic interventions, most studies focus on what interventions are effective for patients, research on the proper understanding and use of such tools are insufficient [14].

Van Peppen et al. [15] has reported that lack of time and burden of cost in the clinical setting is an impediment to the use of assessment tools. Other studies have reported that a lack of knowledge about the assessment tools is a major impediment to their use [16]. In addition, the floor and ceiling effects of the assessment tools are difficult to assess for a variety of patients, and evaluating with use of equipment also has limitations in terms of space, securing professional manpower, and equipment being expensive [12,17]. Due to these barriers, it is not easy for therapists to perform a clinical assessment of the patients' functional level [18]. However, there is no research on the domestic physical therapists' perception of the various gait assessment tools, the frequency of the tools being used, and the barriers in the use of these tools tools that may be imposed onto the therapists.

Therefore, the purpose of this study was to investigate the perception of gait assessment tools by physical therapists, the frequency of its use, and to investigate the barriers that may affect the evaluation process. In this way, physical therapists will provide the basic data for applying effective assessment tools for gait evaluation.

\section{Methods}

\section{Subject and procedure}

Physical therapists from general hospitals, hospice, public health centers, and physical therapy seminars from the Seoul, Gyeonggi area who understood and agreed to the study procedures were included in the study.

After receiving permission to use the survey used from a study by Salbach et al. [16], the Korean translation process was commenced in December of 2015, and after the survey was examined by 11 physical therapists within the same year, the questionnaire was revise. In January 2016, the study was approved by the ethics committee of Sahmyook University (IRB No. SYUIRB2015-098). The purpose of this study was to investigate the physical therapists' perception of the gait assessment tools from the Seoul and Gyeonggi area from March 20, 2016 to July 22, 2016 through a cross-sectional study with use of a self-filling questionnaire consisting of 35 items. The distribution of the questionnaires were either performed at a physical therapy seminar, or after receiving an informed consent by phone, the questionnaires were sent and retrieved by mail, or they were personally distributed and retrieved through personal visits to the hospital where the therapists were located. From a total of 700 questionnaires that had been distributed, 350 questionnaires (50\%) were retrieved, however, with the exclusion of 140 questionnaires (20\%), the remaining 210 questionnaires $(30 \%)$ were used for analysis.

\section{Survey instrument}

The questionnaire consisted of eight items that asked about general characteristics, such as gender, age, level of education, clinical experience, treatment field, number of daily patient treatments, name of institution, and institution

Table 1. Verification of reliabilty

\begin{tabular}{|c|c|c|c|c|}
\hline Field & Content & Item no. & $\begin{array}{c}\text { No. of } \\
\text { question }\end{array}$ & Reliability \\
\hline Individual assessment tool frequency of its use & Use of the 10 gait assessment tools & II. $1-10$ & 10 & 0.811 \\
\hline Perception of individual assessment tools & Perception of the 10 gait assessment tools & III. $1-10$ & 10 & 0.901 \\
\hline
\end{tabular}


location (Supplementary material). The standardized gait assessment tools that were in question for their recognition amongst the therapists were the 10MWT [19], 6MWT [19], TUG [20], DGI [21], RMI [22], FAC [23], MAS [19], FIM [19], Chedoke-McMaster stroke assessment [19], instrumented measurement tools [12]. The questionnaire consisted of a total of 35 items, which included 10 items on perception of the measures, 10 items on the frequency of the assessment tools used, three items asking about the comprehensive perception of the assessment tool, four items asking about the barriers imposed in using the gait assessment tool.

The 5-point Likert scale was used to answer the questionnaire.

In order to test the internal consistency of the questionnaire items, the reliability coefficient Cronbach $\alpha$ was calculated as shown in Table 1.

\section{Data analysis}

All data was analyzed with use of IBM SPSS Statistics ver. 21.0 (IBM Co., Armonk, NY, USA). A frequency analysis was performed to normalize the general characteristics of subjects' perception and frequency of use of the gait assessment tools, and the obstacle factors imposed on gait assessment.

\section{Results}

The general characteristics of the respondents are shown in Table 2. In the evaluation of the perception of the individual gait assessment tools, the TUG was found to be the most useful ( $\mathrm{n}=153,72.9 \%$ ), and the therapists showed high perception and understanding of the 10MWT $(\mathrm{n}=149$, $71.0 \%)$ and the $6 \mathrm{MWT}(\mathrm{n}=123,58.6 \%)$.

On the contrary, only 56 therapists were aware of the Chedoke-McMaster stroke assessment $(\mathrm{n}=56,26.7 \%$; Table 3).

Table 2. General characteristics of subjects

$(\mathrm{N}=210)$

\begin{tabular}{llr}
\hline \multicolumn{1}{c}{ Background factor } & \multicolumn{1}{c}{ Classification } & Respondents \\
\hline Gender & Male & $117(55.7)$ \\
& Female & $93(44.3)$ \\
Age (yr) & $<30$ & $121(57.6)$ \\
& $30-39$ & $68(32.4)$ \\
Educational level & $\geq 40$ & $21(10.0)$ \\
& Diploma & $39(18.6)$ \\
& Bachelor's & $145(69.0)$ \\
Clinical experience (yr) $)$ & Graduated school & $26(12.4)$ \\
& $<5$ & $110(52.4)$ \\
& $5-10$ & $64(30.5)$ \\
Daily therapy session & $>10$ & $36(17.1)$ \\
& $<10$ & $31(14.8)$ \\
& $10-14$ & $144(68.6)$ \\
Institution type & $\geq 15$ & $35(16.7)$ \\
& Tertiary hospital & $38(18.1)$ \\
& General hospital & $24(11.4)$ \\
& Hospital & $108(51.4)$ \\
Location & Others & $40(19.0)$ \\
Total & Seoul & $165(78.6)$ \\
& Gyeonggi & $45(21.4)$ \\
& & $210(100)$ \\
\hline
\end{tabular}

Values are presented as number (\%).

The sum of the percentages does not equal $100 \%$ because of rounding.

Table 3. Perception of individual gait assessment tools

$(\mathrm{N}=210)$

\begin{tabular}{|c|c|c|c|c|}
\hline Gait assessment tool & High & Medium & Low & Total \\
\hline 10 meters walk test & $149(71.0)$ & $50(23.8)$ & $11(5.2)$ & $210(100)$ \\
\hline 6-minute walk test & $123(58.6)$ & $70(33.3)$ & $17(8.1)$ & $210(100)$ \\
\hline TUG & $153(72.9)$ & $48(22.9)$ & $9(4.3)$ & $210(100)$ \\
\hline DGI & $86(41.0)$ & $94(44.8)$ & $30(14.3)$ & $210(100)$ \\
\hline RMI & $58(27.6)$ & $115(54.8)$ & $37(17.6)$ & $210(100)$ \\
\hline FAC & $87(41.4)$ & $97(46.2)$ & $26(12.4)$ & $210(100)$ \\
\hline MAS & $98(46.7)$ & $88(41.9)$ & $24(11.4)$ & $210(100)$ \\
\hline FIM & $88(41.9)$ & $101(48.1)$ & $21(10.0)$ & $210(100)$ \\
\hline Chedoke-McMaster stroke assessment & $56(26.7)$ & $116(55.2)$ & $38(18.1)$ & $210(100)$ \\
\hline Instrumented measurement tools & $115(54.8)$ & $76(36.2)$ & $19(9.0)$ & $210(100)$ \\
\hline Total & $101(48.0)$ & $86(41.0)$ & $23(11.0)$ & $210(100)$ \\
\hline
\end{tabular}

Values are presented as number (\%).

The sum of the percentages does not equal $100 \%$ because of rounding.

TUG: timed up and go test, DGI: dynamic gait index, RMI: rivermead mobility index, FAC: functional ambulation classification, MAS: motor assessment scale, FIM: functional independent measure. 
In regards to comprehensive perception of the assessment tools, 89 therapists (42.4\%) answered that the gait assessment tools were useful when asked the question, "Do you think standardized gait measures quantify for the severity of a gait disorder?" For the question, "Are standardized gait measures suitable for assessing walking ability of mild, moderate, or severe patients?", 72 therapists $(34.3 \%)$ considered the gait assessment tools to be fit and suitable. For the final question that asked "Are the clinical practice guidelines suggesting for special assessment tools for evaluating or monitoring walking ability?" 40 therapists (19.0\%) answered 'highly agree' or 'agree' while 48 therapists (22.9\%) answered 'highly disagree' or 'disagree' (Table 4).

The respondents answered that they had always or frequently used the TUG $(\mathrm{n}=116,55.2 \%), 10 \mathrm{MWT}(\mathrm{n}=100$, $47.6 \%)$ and 6MWT $(\mathrm{n}=51,24.3 \%)$. In contrast, only 4 $(1.9 \%)$ respondents stated that they used the ChedokeMcMaster stroke assessment and RMI (Table 5). From the questions asking about the barriers imposed on the use of gait assessment tools within the clinic, lack of time was shown to be the greatest obstacle.

\section{Discussion}

Gait pathologies have a negative impact on the performance of daily life activities and social participation, which lowers quality of life [2]. Physical therapy can play a major role in addressing gait pathologies [3-6] and therefore, performance of an accurate gait assessment is a priority in developing effective gait interventions [7]. Therefore, the purpose of this study was to investigate the physical therapists' perception and frequency of the gait measures used, the barriers imposed on the use of the gait measures, and to provide basic data for effective application of the measures. In regards to perception of the gait assessment tools, the therapists were the most aware of the functional performance tests for gait, such as the TUG, 10MWT, and 6MWT. In contrast, there was a variety of perception rates for the assessment tools based on an ordinal scale, such as the DGI $(n=86$, $41.0 \%)$, RMI $(\mathrm{n}=58,27.6 \%)$, FAC $(\mathrm{n}=87,41.4 \%)$, MAS

Table 4. Comprehensive perception of gait measurement

$(\mathrm{N}=210)$

\begin{tabular}{|c|c|c|c|c|}
\hline Comprehensive perception of gait measurement & High & Medium & Low & Total \\
\hline Do you think standardized gait measures quantify for the severity of a gait disorder? & $89(42.4)$ & $102(48.6)$ & $19(9.0)$ & $210(100)$ \\
\hline $\begin{array}{l}\text { Are standardized gait measures suitable for assessing walking ability of mild, } \\
\text { moderate, or severe patients? }\end{array}$ & $72(34.3)$ & $114(54.3)$ & $24(11.4)$ & $210(100)$ \\
\hline $\begin{array}{l}\text { Are the clinical practice guidelines suggesting for special assessment tools for } \\
\text { evaluating or monitoring walking ability? }\end{array}$ & $40(19.0)$ & $122(58.1)$ & $48(22.9)$ & $210(100)$ \\
\hline Total & $67(31.9)$ & $113(53.8)$ & $30(14.3)$ & $210(100)$ \\
\hline
\end{tabular}

Values are presented as number $(\%)$.

Table 5. Use of individual gait assessment tools

$(\mathrm{N}=210)$

\begin{tabular}{lcrrr}
\hline \multicolumn{1}{c}{ Gait measure } & High & Medium & Low & Total \\
\hline 10 meters walk test & $100(47.6)$ & $44(21.0)$ & $66(31.4)$ & $210(100)$ \\
6-minute walk test & $51(24.3)$ & $34(16.2)$ & $125(59.5)$ & $210(100)$ \\
TUG & $116(55.2)$ & $34(16.2)$ & $60(28.6)$ & $210(100)$ \\
DGI & $22(10.5)$ & $25(11.9)$ & $163(77.6)$ & $210(100)$ \\
RMI & $4(1.9)$ & $12(5.7)$ & $194(92.4)$ & $210(100)$ \\
FAC & $44(21.0)$ & $15(7.1)$ & $151(71.9)$ & $210(100)$ \\
MAS & $50(23.8)$ & $29(13.8)$ & $131(62.4)$ & $210(100)$ \\
FIM & $25(11.9)$ & $26(12.4)$ & $159(75.7)$ & $210(100)$ \\
Chedoke-McMaster stroke assessment & $4(1.9)$ & $11(5.2)$ & $195(92.9)$ & $210(100)$ \\
Instrumented measurement tools & $20(9.5)$ & $22(10.5)$ & $168(80.0)$ & $210(100)$ \\
Total & $44(21.0)$ & $25(11.9)$ & $141(67.1)$ & $210(100)$ \\
\hline
\end{tabular}

Values are presented as number (\%).

The sum of the percentages does not equal $100 \%$ because of rounding.

TUG: timed up and go test, DGI: dynamic gait index, RMI: rivermead mobility index, FAC: functional ambulation classification, MAS: motor assessment scale, FIM: functional independent measure. 
( $\mathrm{n}=98,46.7 \%)$, FIM ( $\mathrm{n}=88,41.9 \%)$, Chedoke-McMaster stroke assessment $(n=56,26.7 \%)$ as presented in Table 3. Therefore, the Chedoke-McMaster stroke assessment and RMI had a low perception rate (recognition) compared to the DGI, FAC, MAS, FIM.

Although the comprehensive perception of the assessment tools showed that the existing tools used for gait assessment was useful for treating patients, the assessment tools showed a lower perception of the patient's degree of injury or evaluating all other aspects of gait (Table 4). This may be due to the evaluation category of a single assessment tool is limited. To improve this, it is necessary to use a wide range of evaluation tools. Similar to how the Postural Assessment Scale for Stroke and Activities-specific Balance Confidence is used to address the limitations of the Berg Balance Scale, the use of several assessment tools is more effective rather than use of a single assessment tool [17,24]. Subsequently, 40 respondents (19.0\%) answered 'highly agree' or 'agree' and 48 respondents (22.9\%) answered 'highly disagree' or 'disagree' to the question, "Are the clinical practice guidelines suggesting for special assessment tools for evaluating or monitoring walking ability?" Therefore, although there is a need for clinical practice guidelines to suggest for assessment tools to evaluate gait ability or to monitor gait, they are currently still lacking $[14,16]$.

Rather than the use of ordinal scale-based assessment tools, functional performance tests, such as the TUG, 10MWT, and 6MWT are the most frequently used apparently due to its simplicity and time limitation imposed within the clinic (Table 5).

In addition, although the gait assessment tools are considered useful, the evaluation status is low, which is affected by the clinical environment (Tables 3, 5). A previous study has shown that the Chedoke-McMaster stroke assessment $(61.1 \%)$ is the most commonly used assessment tool [16], however, this study only showed a rate of $1.9 \%$. It appears that different curricula and health care systems across the countries have an effect on the use of the assessment tools $[8,25]$. Lack of time was considered to serve as the largest obstacle in the use of gait assessment tools. Of the general characteristics of this study, 144 therapists $(68.6 \%)$ had 10 -14 daily therapy sessions, 35 therapists (16.7\%) had 15 daily or more daily therapy sessions, and 179 therapists (85.2\%) had over 10 daily therapy sessions while a Canadian study reported that 291 therapists $(78.9 \%)$ had over 10 daily therapy sessions, which demonstrates how busy the domestic clinical environment is [26]. A busy, domestic clinical en- vironment may have influenced the selection of gait assessment tools and could be an important indicator of the different clinical circumstances across the countries

This study has shown that the therapists were the most highly aware of the functional performance tests, such as the TUG, 10MWT, and 6MWT and that the lack of time posed as the greatest obstacle in use of gait assessment tools. This result may be used as a basis for effective gait assessment tool application.

This study has some limitations. It is difficult to generalize the results of this study through only analysing the responses from 210 physical therapists in the Seoul and Gyeonggi area. Out of the numerous gait assessment tools available, this study only selected 10 gait assessment tools for analysis purposes. It is difficult to exclude subjectivity and bias of the individual since this research was conducted through self-filling questionnaires. In addition, it is difficult to obtain statistical results by simple analysis of frequency.

Further studies should consider factors that may affect the therapists' decision making in gait measures and eliminate the barriers in the use of those measures. In addition, there is a need for comparison of the use of gait measures from other countries as well as a guideline for the use of an appropriate measure within the clinic.

\section{Conflict of Interest}

The authors declared no potential conflicts of interest with respect to the authorship and/or publication of this article.

\section{Supplementary Materials}

Supplementary data can be found with this article online at https://doi.org/10.14474/ptrs.2017.6.2.90.

\section{References}

1. Perry J. Gait analysis: normal and pathological function. Thorofare, NJ: Slack; 1992.

2. Mayo NE, Wood-Dauphinee S, Côté R, Durcan L, Carlton J. Activity, participation, and quality of life 6 months poststroke. Arch Phys Med Rehabil 2002;83:1035-42.

3. Langhorne P, Coupar F, Pollock A. Motor recovery after stroke: a systematic review. Lancet Neurol 2009;8:741-54.

4. Sidaway B, Anderson J, Danielson G, Martin L, Smith G. Effects of long-term gait training using visual cues in an individual with Parkinson disease. Phys Ther 2006;86:186-94.

5. Behrman AL, Lawless-Dixon AR, Davis SB, Bowden MG, Nair 
P, Phadke C, et al. Locomotor training progression and outcomes after incomplete spinal cord injury. Phys Ther 2005;85:1356-71.

6. Hornby TG, Zemon DH, Campbell D. Robotic-assisted, bodyweight-supported treadmill training in individuals following motor incomplete spinal cord injury. Phys Ther 2005;85:52-66.

7. Best R, Begg R. Overview of movement analysis and gait features. In: Begg R, Palaniswami M, editors. Computational intelligence for movement science: neural networks and support vector machines, and other emerging technologies. Hershey, PA: Idea Group Inc.; 2006. p. 1-69.

8. Pattison KM, Brooks D, Cameron JI, Salbach NM. Factors influencing physical therapists' use of standardized measures of walking capacity poststroke across the care continuum. Phys Ther 2015;95:1507-17.

9. American Physical Therapy Association. Guide to physical therapist practice. 2nd ed. Alexandria, VA: American Physical Therapy Association; 2001. p. 9-746.

10. Tyson S, DeSouza L. A systematic review of methods to measure balance and walking post-stroke. Part 1: ordinal scales. Phys Ther Rev 2002;7:173-86.

11. Tyson S, DeSouza L. The measurement of balance and walking post-stroke. Part 2: functional performance tests. Phys Ther Rev 2002;7:187-91.

12. Kim BO. Methods in clinical gait analysis. J Korean Acad Rehabil Med 1994;18:191-202.

13. Berg KO, Maki BE, Williams JI, Holliday PJ, Wood-Dauphinee SL. Clinical and laboratory measures of postural balance in an elderly population. Arch Phys Med Rehabil 1992;73:1073-80.

14. Kang TW, Oh DW. Literature review of walking performance tests for people with post-stroke hemiparesis. Korean J Neural Rehabil 2013;3:28-43.

15. Van Peppen RP, Maissan FJ, Van Genderen FR, Van Dolder R, Van Meeteren NL. Outcome measures in physiotherapy management of patients with stroke: a survey into self-reported use, and barriers to and facilitators for use. Physiother Res Int 2008;13:
255-70.

16. Salbach NM, Guilcher SJ, Jaglal SB. Physical therapists' perceptions and use of standardized assessments of walking ability post-stroke. J Rehabil Med 2011;43:543-9.

17. Sibley KM, Salbach NM. Applying knowledge translation theory to physical therapy research and practice in balance and gait assessment: case report. Phys Ther 2015;95:579-87.

18. Grimmer K, Bialocerkowski A, Kumar S, Milanese S. Implementing evidence in clinical practice: the 'therapies' dilemma. Physiotherapy 2004;90:189-94.

19. Finch E, Brooks D, Stratford PW, Mayo NE; Canadian Physiotherapy Association. Physical rehabilitation outcome measures: a guide to enhanced clinical decision making. 2nd ed. Baltimore: Lippincott, Williams \& Wilkins; 2002.

20. Podsiadlo D, Richardson S. The timed "Up \& Go": a test of basic functional mobility for frail elderly persons. J Am Geriatr Soc 1991;39:142-8.

21. Shumway-Cook A, Woollacott MH. Motor control: theory and practical applications. Baltimore: Williams \& Wilkins; 1995.

22. Collen FM, Wade DT, Robb GF, Bradshaw CM. The rivermead mobility index: a further development of the rivermead motor assessment. Int Disabil Stud 1991;13:50-4.

23. Holden MK, Gill KM, Magliozzi MR. Gait assessment for neurologically impaired patients. Standards for outcome assessment. Phys Ther 1986;66:1530-9.

24. Blum L, Korner-Bitensky N. Usefulness of the Berg balance scale in stroke rehabilitation: a systematic review. Phys Ther 2008;88:559-66.

25. Kim K, Park ES, Cho YH, Cho JS, Yu JE, Park RJ, et al. A study on the development of standard curriculum for physical therapy in Korea. J Korean Soc Phys Ther 2006;18:23-32.

26. Sibley KM, Straus SE, Inness EL, Salbach NM, Jaglal SB. Clinical balance assessment: perceptions of commonly-used standardized measures and current practices among physiotherapists in Ontario, Canada. Implement Sci 2013;8:33. 